Published in final edited form as:

Curr Treat Options Oncol. ; 19(11): 55. doi:10.1007/s11864-018-0575-4.

\title{
Sentinel Lymph Node Biopsy and Completion Lymph Node Dissection for Melanoma
}

\author{
Sabran J. Masoud, BS ${ }^{1}$, Jennifer A. Perone, MD $^{2}$, Norma E. Farrow, MD ${ }^{1}$, Paul J. Mosca, \\ MD, PhD, MBA ${ }^{1}$, Douglas S. Tyler, MD ${ }^{2}$, Georgia M. Beasley, MD, MHS ${ }^{1,3,{ }^{*}}$ \\ ${ }^{1}$ Department of Surgery, Duke University, Durham, NC, 27710, USA \\ ${ }^{2}$ Department of Surgery, University Texas Medical Branch, Galveston, TX, USA \\ ${ }^{3}$ Duke University Medical Center, DUMC Box 3118, Durham, NC, 27710, USA
}

\section{Opinion statement}

This review critically evaluates recent trials which have challenged the practice of completion lymph node dissection (CLND) for melanoma patients diagnosed with regional metastasis by positive sentinel lymph node biopsy (SLNB). Two trials in the last 2 years, DeCOG-SLT and MSLT-II, found no significant differences in melanoma-specific survival between patients, whether they received immediate CLND or observation after positive SLNB, despite decreases in nodal recurrence achieved by dissection. These trials together disfavor routine CLND in most patients after positive SLNB. However, their conclusions are limited by study populations which overall harbored a lower burden of SLN disease. Special attention needs to be given to patients who do have higher risk disease, with SLN tumor burdens exceeding $1 \mathrm{~mm}$ in diameter, for whom CLND may remain both prognostic and therapeutic. Current guidelines thus recommend either CLND or careful observation after positive SLNB after appropriate risk stratification of patients. While a decline in CLND is inevitable, treatment of stage III melanoma is witnessing the concurrent rise of effective adjuvant therapies. PD-1 inhibitors such as nivolumab, or combination BRAF/MEK inhibitors for V600E or K mutant melanoma, which were previously available to only trial patients with completely resected stage III disease, are now approved for use in patients with positive SLNB alone. Providers are better equipped than ever to treat clinically occult, regional metastatic disease with SLNB followed by adjuvant therapy for most patients, but should take steps to avoid undertreatment of high-risk patients who may proceed to disease relapse or progression.

\section{Keywords}

Melanoma; Sentinel lymph node biopsy; Lymph node dissection; Staging; Metastasis; Survival

\footnotetext{
*Duke University Medical Center, DUMC Box 3118, Durham, NC, 27710, USA, Georgia.beasley@ duke.edu. Compliance with Ethical Standards

Conflict of Interest

Paul J. Mosca declares that he has no conflict of interest. Douglas S. Tyler declares that he has no conflict of interest. Georgia M. Beasley declares that she has no conflict of interest.

Human and Animal Rights and Informed Consent

This article does not contain any studies with human or animal subjects performed by any of the authors.
} 


\section{Introduction}

Malignant melanoma is the fifth most common cause of cancer, with an incidence that has risen from 7.9 per 100,000in 1975 to 25.8 per 100,000 in 2015 [1]. Despite upward trends in cancer-related survival, an estimated 9320 individuals are expected to die of melanoma in 2018 within the USA alone [2]. Since 2011, there have been rapid advances in systemic melanoma therapy for both the adjuvant and metastatic setting [3]. The surgical management of regional metastasis to the lymph nodes, which predicts a combined 35\% decrease in 5year survival relative to localized disease, has also continued to evolve [2]. Over the past 30 years, surgeons have witnessed a shift from elective lymph node dissection to completion lymph node dissection (CLND) after positive sentinel lymph node biopsy (SLNB) and now toward SLNB alone [4]. The ongoing debate over treating nodal disease hinges on whether removing tumor involved lymph nodes improves melanoma-specific survival or merely predicts a poor melanoma prognosis. Given the higher rate of wound complications and neuropathic pain associated with lymphade-nectomy, cases in which regional and systemic disease concurrently develop, and the advent of effective systemic therapies, recent trials have challenged aggressive regional control with CLND for all patients with a positive SLNB $[5 \bullet \bullet, 6,7 \bullet \bullet, 8,9]$. Here, we discuss current surgical strategies for the management of regional lymph nodes in melanoma.

\section{Sentinel lymph node biopsy}

In an era pre-dating sentinel lymph node biopsy, elective lymph node dissection conferred long-term survival (15 to 20 years) in $25 \%$ of patients, at the cost of increased morbidity and wound complications [10-15]. The application of sentinel lymph node biopsy (SLNB) to malignant melanoma in 1992 by Morton established the predilection of regional metastasis for select lymph nodes, those which received patent blue dye injected near the cutaneous primary lesion [16]. Morton identified tumor in 18\% of these "sentinel" lymph nodes (SLN) compared to only $0.06 \%$ of non-sentinel lymph nodes (NSLN) [16]. The addition of preoperative radiographic lymphoscintigraphy to intraoperative dye mapping saw the accuracy of the procedure rise to $96 \%$, with tumor-positive sentinel nodes outnumbering positive non-sentinel nodes by as much as 11 to 1 [17-21]. In up to $81.8 \%$ of patients, these nodes comprised the sole focus of tumor, solidifying the notion of step-wise nodal disease progression [21].

Among patients with clinical stage I and II melanoma, metastasis to the SLN has proven to be the strongest prognostic factor, with evaluation via SLNB having since been well established in the management of $\mathrm{T} 1 \mathrm{~b}$ through $\mathrm{T} 4$ primary cutaneous melanoma $[4,22,23$, 24•, 25]. The multi-center selective lymphadenectomy trial 1 (MSLT-1) sought to validate the therapeutic, in addition to prognostic, benefit of using SLNB to guide completion lymph node dissection (CLND) [26]. Investigators randomized patients undergoing wide excision to receive either SLNB with immediate CLND for positive findings or observation with interval CLND for nodal relapse. There was no difference in 10-year melanoma specific survival between the 2 arms [26]. However, SLNB improved the 10-year rate of disease-free survival in patients with intermediate-thickness $(1.2$ to $3.5 \mathrm{~mm})$ melanoma and overall 
survival was improved when lymph node metastases were identified by SLNB rather than by clinical or radiographic findings [26]. Thus, removal of microscopic disease accomplished by SLNB may confer a survival advantage for some patients with intermediate thickness melanoma.

A recent American Society of Clinical Oncology (ASCO) and Society of Surgical Oncology (SSO) clinical practice guideline reviewed multiple studies to determine current indications for SLNB [4]. While these guidelines recommend against routine SLNB for patients with thin T1a melanoma ( $<0.8 \mathrm{~mm}$ Breslow depth and non-ulcerated), given the low rate of positivity, they suggest that SLNB should be considered in T1b melanoma ( 0.8 to $1.0 \mathrm{~mm}$ or $<0.8 \mathrm{~mm}$ thick with ulceration) and intermediate thickness T2 or T3 melanoma (1.0 to 4.0 $\mathrm{mm}$ ) [4, class II-III]. SLNB may be performed for patients with thick T4 melanoma (> 4.0 $\mathrm{mm}$ ), despite not yielding significant differences in disease outcome relative to observation [4, class III]. Additionally, as clinically node negative patients have not been studied in many recent clinical trials, stage IIC patients are largely ineligible for adjuvant therapy despite representing a group at high risk for recurrence. Although SLNB may not have historically improved disease outcomes for such patients with thick primary tumors, a positive SLNB in the current era renders these individuals eligible for adjuvant systemic therapies which can successfully mitigate disease recurrence $[27 \bullet, 28 \bullet]$.

While the data have reinforced the clinical benefit of performing SLNB, its role in guiding CLND has been more controversial. Previously, the SLN was assumed to represent the entire nodal basin, and that CLND after positive SLNB would eliminate remaining disease of the non-sentinel lymph nodes (NSLN) to improve regional control [26, 29, 30]. In the largest series of patients with a positive SLN undergoing CLND, including over 1500 individuals, NSLN status was an independent prognostic factor for melanoma specific survival [31-34]. However, subsequent data revealed that only $12-20 \%$ of patients harbor tumor-positive NSLN at the time of CLND [22, 23, 24•]. Based on these results, two large randomized controlled trials, DeCOG-SLT and MSLT-II, were recently completed in to assess the value

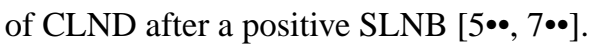

\section{Completion lymph node dissection}

\section{Trials assessing therapeutic benefit: DeCOG-SLT and MSLT-II}

The German Dermatologic Cooperative Oncology Group (DeCOG-SLT) was the first of these two studies, a multi-center, randomized, phase 3 clinical trial evaluating whether CLND resulted in increased survival compared to observation alone in patients with positive SLNB [7••, class II]. Patients were accrued from 41 different German skin cancer centers, including those with primary cutaneous melanoma of the torso, arms, or legs with tumor thickness of at least $1 \mathrm{~mm}$ and micrometastasis in the sentinel lymph node. This study excluded patients with head and neck melanoma, or those with satellite, in-transit, regional, or distant metastatic disease. Patients were randomized into equal groups and stratified by primary tumor thickness, ulceration of the primary tumor, and intent to initiate adjuvant interferon therapy. Patients were accrued between January 1, 2006 and December 1, 2014. Investigators did raise concerns for low statistical power to detect hypothesized betweengroup differences in the primary endpoint of distant metastasis-free survival (DMFS), due to 
both overestimating rates of distant metastases and achieving half the expected accrual to the CLND arm [7••]. Despite low enrollment and early trial closure, investigators enrolled 483 patients (241 to observation and 242 to the CLND group).

There were no significant differences between groups with regard to gender, median age at diagnosis, body site, primary tumor characteristics (Breslow thickness, presence of ulceration, histologic criteria), or size of metastasis in the SLNB specimen [7••]. Both groups were followed for 3 years (median follow-up of 35.5 and 33 months respectively for observation and CLND groups). All patients received a physical exam, lymph node ultrasound, and blood tests (serum S100b) every 3 months, as well as imaging every 6 months (whole body CT scan, MRI, or PET-CT).

In the intention-to-treat analysis, there was no significant difference between groups with respect to DMFS [7••]. At 3 years, DMFS for the observation group was 77\% (90\% CI 71.982.1 ) and $74.9 \%$ (90\% CI 69.5-80) for the CLND group (HR 1.03, 90\% CI 0.71-1.5, $P=$ 0.87) [7••]. Between CLND and observation, investigators also found no differences in the secondary endpoints of 3-year recurrence-free survival (RFS) or overall survival (OS) (66.8\% vs. $67.4 \%, P=0.75$ and $81.2 \%$ vs. $81.7 \%, P=0.87$ ) [7••]. In a multivariable proportional hazards regression analysis, SLN tumor burden and tumor thickness were independent predictors of DMFS, OS, and RFS [7••]. Adverse events-including lymphedema, lymphatic fistula, seroma, infection, and delayed wound healing-were observed in $24 \%$ of patients who received CLND, with $8 \%$ reporting grade 4 events [7••].

Recently, 5-year follow-up results were reported at ASCO 2018 [35••]. At a median followup time of 72 months, there continued to be no significant difference in DMFS between the observation (68\%) and CLND (65\%) groups (HR 1.08, 90\% CI 0.74-1.39, $P=0.65$ ) [35••]. Likewise, neither observation nor CLND was superior with regard to RFS (HR 1.01, 90\% CI $0.8-1.28, P=0.94$ ), OS (HR $0.99,90 \%$ CI $0.74-1.31, P=0.93$ ), or cumulative rates of regional lymph node metastasis $(16.3 \%$ vs. $10.8 \%, P=0.11)[35 \bullet \bullet$.

The multi-center selective lymphadenectomy trial 2 (MSLT-II) was an international, randomized, phase 3 clinical trial comparing immediate CLND against observation in melanoma patients positive for sentinel node metastasis [5••, class I]. Investigators chose 3 year melanoma-specific survival (MSS) as the primary outcome, and disease-free survival (DFS), OS, nodal recurrence-free survival, extent of nodal involvement, and DMFS as secondary outcomes. Investigators screened and enrolled 3531 patients aged 18 to 75 years across 63 centers between December 2004 and March 2014. Patients diagnosed with nodal metastasis by either SLNB $(N=377)$ or RT-PCR $(N=226)$, in addition to 1431 patients who directly entered the two study arms, were assigned to receive immediate CLND or observation with regular follow-up and nodal ultrasonography. A total of 1939 patients were randomized under a permuted block design, and stratified by Breslow thickness, ulceration, method of metastasis detection, and enrollment in an MSLT-1 center. Study groups were similar with respect to the above parameters, as well as in age, sex, smoking status, primary tumor location, SLN tumor burden, and receipt of adjuvant therapy [5••]. Patients were evaluated through a median follow-up period of 43 months and analyzed on both a perprotocol and intention-to-treat basis. 
There were no significant differences in either mean 3-year MSS $(86 \pm$ SE $1.3 \%$ SE and 86 $\pm 1.2 \%, P=0.42$ ) or DMFS (HR 1.10, 95\% CI 0.92-1.31, $P=0.31$ ) between patients who underwent CLND or observation, whether lymph node metastasis was detected molecularly by RT-PCR or pathologically via SLNB [5••]. A significant difference was observed in 3year DFS, at $68 \pm 1.7 \%$ in the CLND group, vs. $63 \pm 1.7 \%$ in the observation group ( $P$ $=0.05)[5 \bullet \cdot$. Moreover, NSLN recurrence accrued at a higher rate in patients undergoing observation and was an independent prognostic factor for melanoma-related death (HR 1.78, $P=0.005$ ) [5••]. This stands in contrast to age, sex, location of primary lesion, and number of positive sentinel nodes, none which independently predicted melanoma-related death [5••]. Regarding complications, $24.1 \%$ of MSLT-II participants in the CLND group experienced lymphedema compared to only $6.3 \%$ of the observation (SLNB only) group $[5 \cdot \bullet]$.

MSLT-II largely recapitulated the conclusions of DeCOG-SLT, with no significant differences in 3-year DMFS, OS, or MSS between CLND and observation groups [5••]. Table 1 summarizes similarities and differences between the trials $[5 \bullet \bullet, 7 \bullet \bullet]$. Where the former study was underpowered to detect the hypothesized difference in DMFS between CLND and observation, MSLT-II achieved $83 \%$ power to detect a $5 \%$ difference in 3-year melanoma-specific survival [5••, 7••]. MSLT-II findings also agreed with DeCOG-SLT on the prognostic impact of primary tumor characteristics. Relative to lesions under $1.50 \mathrm{~mm}$ in Breslow depth, mortality hazard was more pronounced in the observation over CLND groups for intermediate thickness $(1.50$ to $3.50 \mathrm{~mm}$ ) lesions (HR 2.46, $P=0.004 \mathrm{vs.} \mathrm{HR}$ 1.64, $P=0.07$ ), as well as thick ( $>3.50 \mathrm{~mm}$ ) lesions (HR 4.32, $P<0.001$ vs. HR 3.82, $P<0.001$ ) [5••]. Ulceration also reflected a poorer prognosis in the observation than the CLND group (HR 2.17, $P<0.001$ vs. HR 1.97, $P<0.001$ ) [5••].

The two studies diverged with respect to disease recurrence. DECOG-SLT revealed no between-group difference in 3-year recurrence-free survival (RFS) [7••]. For patients in the observation arm, MSLT-II established a significant decrease in 3-year disease free survival (DFS) and corresponding higher 3- and 5-year rates for recurrence of any kind compared to the CLND arm (22.9\% and $26.1 \%$ vs. $17.9 \%$ and $19.9 \%)$ [5••]. The results signal a departure from the conclusions of DeCOG-SLT, which could not confidently distinguish the rates of regional nodal recurrence between study arms [7••]. Even when excluding satellite and intransit locoregional recurrence, MSLT-II showed decreased node-only RFS in the observation group compared to the dissection group ( $77 \pm 1.5 \%$ vs. $92 \pm 1.0 \%, P<0.001)$ $[5 \cdot \bullet]$.

\section{The declining role of completion lymphadenectomy}

The above studies sought to address whether CLND is therapeutic, or only provides

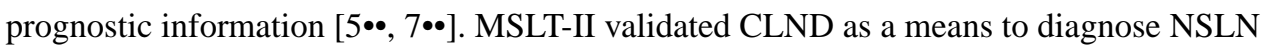
metastasis, which was again shown to be a significant prognostic factor for melanomarelated death [5••]. However, despite achieving a 70\% relative decrease in NSLN recurrence, CLND did not produce an additional survival benefit in patients diagnosed with nodal metastasis [5••]. At present, assessment of NSLN via CLND provides staging and prognostic information, though the therapeutic value of CLND for all patients remains doubtful. 
In addition to challenging the survival benefit of CLND, these data have also called into question the role of CLND in controlling the progression to distant disease. As a corollary to published study findings, supplemental data from MSLT-II showed that while CLND was associated with lower rates of nodal recurrence (e.g., node-only, nodal plus locoregional, or nodal plus distant), CLND had no effect on local or distant recurrences alone [5••]. Coit thus argues that achieving control of nodal tumor deposits beyond the SLN is altogether unrelated to the development of distant metastasis or survival [36•]. Further citing a mere 6.4 percentage-point decrease in node-only relapse achieved by CLND within MSLT-II and the marked increase in CLND-associated complications in both DeCOG-SLT and MSLT-II, Coit concludes that there is sufficient evidence to eschew the practice of CLND after SLNB altogether $[5 \bullet \bullet, 7 \bullet \bullet, 36 \bullet]$.

\section{Lymphadenectomy for high-risk and non-sentinel lymph node disease}

While compelling, calls to abandon CLND for all patients should be qualified. First, it should be noted that MSLT-II patients in the observation group underwent frequent clinical follow-up (visits every 4 months for 2 years; every 6 months between years 3 and 5) and nodal evaluation with ultrasound (annually for 5 years) [5••, 36•]. The authors of MSLT-II stipulate that their recommendation to forego CLND after SLNB was not proven to be safe for all patients, especially when intensive follow-up is unavailable [5••].

Second, failure to establish the advantage of CLND in the general node-positive population does not preclude there being a subset of patients at high risk for non-sentinel lymph node (NSLN) involvement, for whom CLND may provide therapeutic benefit. Investigators have attempted to identify predictors of NSLN recurrence or disease progression, including anatomic location of the primary tumor, and pathologic properties of the SLNB specimen like tumor burden and number of positive nodes [31-33]. Of these, tumor burden has proven most predictive, with SLN tumor deposits greater than $2 \mathrm{~mm}$ in diameter portending NSLN recurrence rates as high as $25 \%$ [37-39]. DeCOG-SLT and MSLT-II evaluated both the quantity and disease burden of tumor-positive sentinel nodes to identify patients at greatest risk for melanoma-related death $[5 \bullet \bullet, 7 \bullet \bullet]$. Neither trial demonstrated a significant

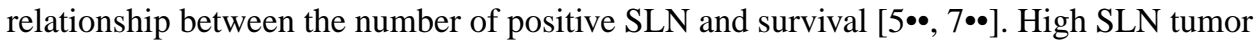
burden, defined as tumor deposits exceeding $1 \mathrm{~mm}$ in diameter, was a significant prognostic factor in multivariable analyses, albeit without reflecting differences between observation and CLND groups in 3-year DMFS or MSS [5••]. Notably, two thirds of patients in both studies had micrometastases of less than or equal to $1 \mathrm{~mm}$ in their SLN and thus comprise a

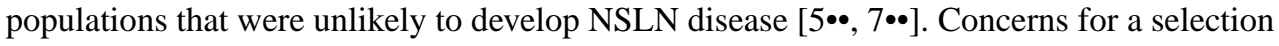
bias against individuals most likely to develop NSLN metastasis led the MSLT-II authors to ultimately limit their recommendations against CLND to patients with SLN tumor deposits less than $1 \mathrm{~mm}[5 \bullet \bullet]$.

In addition to tumor burden, investigators have also explored other immunologic hallmarks of high-risk disease and NSLN recurrence within SLNB specimens, alongside routine histopathologic characteristics. Studies have shown positive correlations between RFS/OS and number of $\mathrm{CD} 3+, \mathrm{CD} 4+$, and CD8+ tumor-infiltrating lymphocytes in tumor-positive sentinel nodes [40•, class IV]. Similarly, elevated levels of regulatory T cell markers like 
FOXP3 and indoleamine 2,3-dioxygenase (IDO), an enzyme implicated in limiting T cell function, have been linked with poorer outcome in terms of local, regional, or distant recurrence [41, class III, 42, class IV]. To date, none of these predictors has been favored by the current NCCN guidelines or 8th edition AJCC Cancer Staging Manual for performing risk stratification following SLNB [25, 43].

\section{Surgical practice implications}

Previous analysis of surgical practice patterns in the National Cancer Database (NCDB) has shown that of the 17,524 patients who underwent SLNB between 2004 and 2005,2942 were positive for SLN metastasis, and half of those (1471) received CLND [44]. The results of DeCOG-SLT and MSLT-II will no doubt lead to a decline in the number of lymphadenectomies performed by surgeons. The treatment of invasive breast cancer witnessed a similar downward trend in axillary lymph node dissections in favor of SLNB, with rates having decreased $2.43 \%$ per year, each year from 2007 to 2014 [45•]. In melanoma, as with invasive breast cancer, the decrease in rates of CLND will likely witness a concomitant increase in the complexity of nodal dissection. Where CLND is performed on clinically negative lymph node basins with normal anatomy, salvage lymphadenectomy for nodal recurrence involves navigating a more complex space often distorted by macrometastatic tumor. For surgical training programs, increased technical difficulty and decreased case volume involving normal anatomy highlight a growing challenge to equip future trainees with the necessary skills to manage recurrent regional melanoma [45•].

\section{Pharmacologic treatment}

\section{Adjuvant therapy in the post-DeCOG-SLT/MSLT-II era}

The last several years have witnessed the approval of multiple systemic therapies for use in the adjuvant setting after oncologic resection of stage III or IV melanoma. Notably, all these recent trials enrolled patients with at least stage III disease that had been resected via CLND after positive SLNB. EORTC 18071, comparing anti-CTLA-4 antibody, ipilimumab, to placebo, demonstrated increased 3-year RFS ( $46.5 \%$ vs. $34.8 \%, P=0.0013)$, OS (65.4\% vs. $54.4 \%, P=0.001)$, and DMFS ( $48.3 \%$ vs. $38.9 \%, P=0.002)$ in patients with resected stage III melanoma [46•, 47•]. However, significant immune-related adverse events (IRAE) in $41.6 \%$ of patients, and double the rate of grade 3-4 toxicity, lead to its discontinuation in half of those treated [46•]. In the Checkmate 238 trial, adjuvant PD-1 inhibition with nivolumab, relative to ipilimumab, was associated with improved 1-year RFS (70.5\% vs. $60.8 \%, P<0.001)$ and lower toxicity ( $14.4 \%$ vs. $45.9 \%$ ) for both stage III and IV melanoma [28•]. Keynote-006 likewise demonstrated improved 1-year RFS for patients receiving pembrolizumab, another PD-1 inhibitor, when compared to ipilimumab (55\% vs. 43\%, $P<$ 0.001) [48•]. Still, other trials have assessed the synergistic effects of combination anti-PD-1 plus low-dose anti-CTLA-4 agents, yielding both increased survival and higher toxicity than monotherapy [49•, 50]. For patients with BRAF mutant melanoma, combination BRAF inhibition with dabrafenib plus MEK inhibition with trametinib has shown particular promise in the adjuvant setting, improving 3-year relapse free survival compared to placebo (58\% vs. $39 \%, P<0.001$ ) [27•]. Although the above trials all required CLND, the adjuvant therapies studied therein are now being widely applied in the clinical setting for Stage IIIA 
disease (SLN with $1 \mathrm{~mm}$ tumor deposits). Moving forward, SLNB positivity can alone qualify patients for treatment. How differences between the original trial populations and the more heterogeneous clinical population translate to relative clinical outcomes after adjuvant therapy is unclear.

Currently, NCCN guidelines favor adjuvant nivolumab monotherapy for resected stage IIIB/C and IV melanoma [25, NCCN category 1 ]. Alternatively, for patients with BRAF V600E or V600 K mutations and SLN tumor burden exceeding $1 \mathrm{~mm}$, combination dabrafenib/trametinib can be used [25, NCCN category 1]. For low-risk sentinel-nodepositive disease (e.g., stage IIIA), NCCN accordingly lists observation as a viable alternative to adjuvant therapy [25, NCCN category $2 \mathrm{~A}]$. Therein lies a continued challenge for providers to balance potential overtreatment of patients whose disease can be sufficiently managed with SLNB, against undertreatment of high-risk patients who may proceed to disease relapse. Much like the decision to perform CLND, the choice to initiate or delay adjuvant therapy would likewise benefit from improved models of risk stratification to better identify patients at risk for NSLN or distant recurrence.

\section{Conclusions}

Sentinel lymph node biopsy has refined previous notions of all-or-nothing regional nodal metastasis to instead favor the step-wise microscopic involvement of sentinel nodes before non-sentinel nodes, and resulted in the adoption of selective over elective lymphadenectomy. When data suggested that biopsy of the sentinel nodes was itself therapeutic, investigators sought to understand whether completion lymphadenectomy was necessary. Recent data from two randomized controlled trials, DeCOG-SLT and MSLT-II, demonstrated that observation yielded comparable overall, disease-specific, and metastasis-free survival to CLND, at the cost of increased non-sentinel lymph node recurrence [5••, $7 \bullet \bullet]$.

At this juncture, the combined data establish that SLNB achieves similar outcomes to CLND with lower morbidity for most patients presenting with clinically occult regional lymph node metastasis. SLNB provides prognostic value, is therapeutic in patients with intermediate thickness melanoma, and can be used to determine eligibility for effective adjuvant therapy. The extant challenge for providers is to define the optimal treatment for patients that are at high risk for developing regional recurrence, and at low risk of developing systemic disease. For these patients, outcomes after prompt removal of NSLN via CLND are less clear. Models incorporating histologic (SLN microscopic tumor burden) and immunologic criteria (relative tumor-infiltrating and regulatory T lymphocyte function) may help guide future prospective studies in predicting nodal recurrence and systemic disease [40•, 41-43]. Presently, patients with suspected NSLN disease that remains isolated to the regional nodal basin may continue to be candidates for CLND. For the majority of patients without suspected NSLN involvement, who harbor low SLN tumor burdens, routine CLND is no longer recommended after positive SLNB.

\section{Acknowledgments}

Sabran J. Masoud received support for travel for the 2018 Melanoma Research Alliance Conference from the PolkaDot Mama Melanoma Research Foundation.

Curr Treat Options Oncol. Author manuscript; available in PMC 2019 August 06. 
Jennifer A. Perone is supported by a Clinical and Translational Science Award Linked Training (TL1) Award from the National Center of Advancing Translational Sciences, National Institutes of Health (NIH) (TL1TR001440).

Norma E. Farrow received support from an NIH T32 grant (T32-CA009111).

\section{References and Recommended Reading}

Papers of particular interest, published recently, have been highlighted as:

- Of importance

•. Of major importance

1. Siegel RL, Miller KD, Jemal A. Cancer statistics, 2018. CA Cancer J Clin. 2018;68(1):7-30. 10.3322/caac.21442. [PubMed: 29313949]

2. Noone AM, Howlader N, Krapcho M, Miller D, Brest A, Yu M, et al., editors. SEER Cancer Statistics Review, 1975-2015 National Cancer Institute https://seer.cancer.gov/csr/1975_2015/. Accessed July 18, 2018.

3. Napolitano S, Brancaccio G, Argenziano G, Martinelli E, Morgillo F, Ciardiello F, et al. It is finally time for adjuvant therapy in melanoma. Cancer Treat Rev. 2018;69:101-11. 10.1016/j.ctrv. 2018.06.003. [PubMed: 29957365]

4. Wong SL, Kennedy EB, Lyman GH. Sentinel lymph node biopsy and management of regional lymph nodes in melanoma: American Society of Clinical Oncology and Society of Surgical Oncology clinical practice guideline update summary. J Oncol Pract. 2018;14(4):242-5. 10.1200/ JOP.2017.028241. [PubMed: 29232158]

5. Faries MB, Thompson JF, Cochran AJ, Andtbacka RH, Mozzillo N, Zager JS, et al. Completion dissection or observation for sentinel-node metastasis in melanoma. N Engl J Med. 2017;376(23): 2211-22. 10.1056/NEJMoa1613210.

•- An international phase 3 randomized controlled clinical trial, studying patients with sentinel-node metastases diagnosed by PCR or SLNB, finding immediate completion lymph node dissection resulted in higher disease-free survival and rates of lymphedema, but no increase in melanomaspecific survival compared to observation.

[PubMed: 28591523]

6. Guggenheim MM, Hug U, Jung FJ, Rousson V, Aust MC, Calcagni M, et al. Morbidity and recurrence after completion lymph node dissection following sentinel lymph node biopsy in cutaneous malignant melanoma. Ann Surg. 2008;247(4):687-93. 10.1097/SLA.0b013e318161312a. [PubMed: 18362633]

7. Leiter U, Stadler R, Mauch C, Hohenberger W, Brockmeyer N, Berking C, et al. Complete lymph node dissection versus no dissection in patients with sentinel lymph node biopsy positive melanoma (DeCOG-SLT): a multicentre, randomised, phase 3 trial. Lancet Oncol. 2016;17(6):757-67.10.1016/ S1470-2045(16)00141-8.

- A multi-center phase 3 randomized controlled clinical trial of patients with cutaneous melanoma of the torso, arms, or legs with positive sentinel lymph node biopsy, showed no difference in survival in patients treated with complete lymph node dissection compared with observation only.

[PubMed: 27161539]

8. Slagelse C, Petersen KL, Dahl JB, Finnerup K, Greene K, Leong SP, et al. Persistent postoperative pain and sensory changes following lymph node excision in melanoma patients: a topical review. Melanoma Res. 2014;24(2):93-8. 10.1097/CMR.0000000000000041. [PubMed: 24346167]

9. Wrightson WR Wong SL, Edwards MJ, Chao C, Reintgen DS, Ross MI, et al. Complications associated with sentinel lymph node biopsy for melanoma. Ann Surg Oncol. 2003;10(6):676-80. [PubMed: 12839853] 
10. Balch CM, Murad TM, Soong SJ, Ingalls AL, Halpern NB, Maddox WA. A multifactorial analysis of melanoma: prognostic histopathological features comparing Clarkś and Breslowś staging methods. Ann Surg. 1978;188(6):732-42. [PubMed: 736651]

11. White RR, Stanley WE, Johnson JL, Tyler DS, Seigler HF. Long-term survival in 2,505 patients with melanoma with regional lymph node metastasis. Ann Surg. 2002;235(6):879-87. [PubMed: 12035046]

12. Sim FH, Taylor WF, Pritchard DJ, Soule EH. Lymph-adenectomy in the management of stage I malignant melanoma: a prospective randomized study. Mayo Clin Proc. 1986;61(9):697-705. [PubMed: 3747613]

13. Veronesi U, Adamus J, Bandiera DC, Brennhovd IO, Caceres E, Cascinelli N, et al. Inefficacy of immediate node dissection in stage 1 melanoma of the limbs. N Engl J Med. 1977;297(12):62730. 10.1056/NEJM197709222971202. [PubMed: 895764]

14. Balch CM, Murad TM, Soong SJ, Ingalls AL, Richards PC, Maddox WA. Tumor thickness as a guide to surgical management of clinical stage I melanoma patients. Cancer. 1979;43(3):883-8. [PubMed: 427730]

15. Balch CM, Soong S, Ross MI, Urist MM, Karakousis CP, Temple WJ, et al. Long-term results of a multi-institutional randomized trial comparing prognostic factors and surgical results for intermediate thickness melanomas (1.0 to $4.0 \mathrm{~mm}$ ). Intergroup Melanoma Surgical Trial. Ann Surg Oncol. 2000;7(2):87-97. [PubMed: 10761786]

16. Morton DL, Wen DR, Wong JH, Economou JS, Cagle LA, Storm FK, et al. Technical details of intraoperative lymphatic mapping for early stage melanoma. Arch Surg. 1992;127(4):392-9. [PubMed: 1558490]

17. Albertini JJ, Cruse CW, Rapaport D, Wells K, Ross M, DeConti R, et al. Intraoperative radiolympho-scintigraphy improves sentinel lymph node identification for patients with melanoma. Ann Surg. 1996;223(2):217-24. [PubMed: 8597518]

18. Miliotes G, Albertini J, Berman C, Heller R, Messina J, Glass F, et al. The tumor biology of melanoma nodal metastases. Am Surg. 1996;62(1):81-8. [PubMed: 8540654]

19. Reintgen D, Cruse CW, Wells K, Berman C, Fenske N, Glass F, et al. The orderly progression of melanoma nodal metastases. Ann Surg. 1994;220(6):759-67. [PubMed: 7986143]

20. Ross MI, Reintgen D, Balch CM. Selective lymphadenectomy: emerging role for lymphatic mapping and sentinel node biopsy in the management of early stage melanoma. Semin Surg Oncol. 1993;9(3):219-23. [PubMed: 8516607]

21. Thompson JF, McCarthy WH, Bosch CM, O’Brien CJ, Quinn MJ, Paramaesvaran S, et al. Sentinel lymph node status as an indicator of the presence of metastatic melanoma in regional lymph nodes. Melanoma Res. 1995;5(4):255-60. [PubMed: 7496161]

22. Balch CM, Gershenwald JE, Soong SJ, Thompson JF, Ding S, Byrd DR, et al. Multivariate analysis of prognostic factors among 2,313 patients with stage III melanoma: comparison of nodal micrometastases versus macrometastases. J Clin Oncol. 2010;28(14):2452-9. 10.1200/JCO. 2009.27.1627. [PubMed: 20368546]

23. Gershenwald JE, Thompson W, Mansfield PF, Lee JE, Colome MI, Tseng CH, et al. Multiinstitutional melanoma lymphatic mapping experience: the prognostic value of sentinel lymph node status in 612 stage I or II melanoma patients. J Clin Oncol. 1999;17(3):976-83. 10.1200/ JCO.1999.17.3.976. [PubMed: 10071292]

24. McMasters KM, Egger ME, Edwards MJ, Ross MI, Reintgen DS, Noyes RD, et al. Final results of the sunbelt melanoma trial: a multi-institutional prospective randomized phase iii study evaluating the role of adjuvant high-dose interferon alfa-2b and completion lymph node dissection for patients staged by sentinel lymph node biopsy. J Clin Oncol. 2016;34(10):1079-86. 10.1200/jco. 2015.63.3776.

- A multi-institutional phase 3 randomized controlled clinical trial comparing adjuvant interferon alfa- $2 \mathrm{~b}$ and completion lymph node dissection, patients with 1 positive sentinel lymph node by RTPCR who underwent observation had 5-year disease-free survival greater than $80 \%$ or less than $20 \%$ with disease relapse.

[PubMed: 26858331] 
25. Network NCC. In: Melanoma (Version 3.2018). https://www.nccn.org/professionals/ physician_gls/pdf/melanoma.pdf. Accessed July 16, 2018.

26. Morton DL, Thompson JF, Cochran AJ, Mozzillo N, Nieweg OE, Roses DF, et al. Final trial report of sentinel-node biopsy versus nodal observation in melanoma. N Engl J Med. 2014;370(7):599_ 609. 10.1056/NEJMoa1310460. [PubMed: 24521106]

27. Long GV, Hauschild A, Santinami M, Atkinson V, Mandala M, Chiarion-Sileni V, et al. Adjuvant dabrafenib plus trametinib in stage III BRAF-mutated melanoma. N Engl J Med. 2017;377(19): 1813-23. 10.1056/NEJMoa1708539.

- In a phase 3 randomized controlled trial in patients w/resected, stage III melanoma and V600E or V600K mutations, oral dabrafenib plus trametinib had higher relapse-free survival and distant metastasis-free survival compared to placebo.

[PubMed: 28891408]

28. Weber J, Mandala M, Del Vecchio M, Gogas HJ, Arance AM, Cowey CL, et al. Adjuvant nivolumab versus ipilimumab in resected stage III or IV melanoma. N Engl J Med. 2017;377(19): 1824-35. 10.1056/NEJMoa1709030.

-A phase 3 randomized controlled trial comparing adjuvant Nivolumab and Ipilimumab in Resected Stage III or IV Melanoma, finding increased 12-month RFS for nivolumab with a lower rate of grade 3 or 4 adverse events.

[PubMed: 28891423]

29. Wong SL, Morton DL, Thompson JF, Gershenwald JE, Leong SP, Reintgen DS, et al. Melanoma patients with positive sentinel nodes who did not undergo completion lymphadenectomy: a multiinstitutional study. Ann Surg Oncol. 2006;13(6):809-16. 10.1245/ASO.2006.03.058. [PubMed: 16604476]

30. Wong SL, Hurley P, Lyman GH. Sentinel lymph node biopsy for melanoma: American Society of Clinical Oncology and Society of Surgical Oncology joint clinical practice guideline. J Oncol Pract. 2012;8(4):e65-e6. 10.1200/JOP.2012.000676. [PubMed: 29452545]

31. Dewar DJ, Newell B, Green MA, Topping AP, Powell BW, Cook MG. The microanatomic location of meta-static melanoma in sentinel lymph nodes predicts nonsentinel lymph node involvement. $\mathrm{J}$ Clin Oncol. 2004;22(16):3345-9. 10.1200/JCO.2004.12.177. [PubMed: 15310779]

32. Cadili A, Scolyer RA, Brown PT, Dabbs K, Thompson JF. Total sentinel lymph node tumor size predicts nonsentinel node metastasis and survival in patients with melanoma. Ann Surg Oncol. 2010;17(11):3015-20. 10.1245/s10434-010-1145-6. [PubMed: 20552405]

33. McMasters KM, Wong SL, Edwards MJ, Chao C, Ross MI, Noyes RD, et al. Frequency of nonsentinel lymph node metastasis in melanoma. Ann Surg Oncol. 2002;9(2):137-41. [PubMed: 11888869]

34. Pasquali S, Mocellin S, Mozzillo N, Maurichi A, Quaglino P, Borgognoni L, et al. Nonsentinel lymph node status in patients with cutaneous melanoma: results from a multi-institution prognosticstudy. J Clin Oncol. 2014;32(9):935-41. 10.1200/jco.2013.50.7681. [PubMed: 24516022]

35. Leiter UM, Stadler R Mauch C, Hohenberger W, Brockmeyer N, Berking C, et al. Final analysis of DECOG-SLT trial: Survival outcomes of complete lymph node dissection in melanoma patients with positive sentinel node. J Clin Oncol. 2018;36(15_suppl):9501 10.1200/JCO. 2018.36.15_suppl.9501.

-• Three year update of DeCOG-SLT clinical trial showing that after median follow-up of 72 months, no survival benefit was observed in melanoma patients with positive SLNB undergoing CLND compared to observation.

36. Coit D The enigma of regional lymph nodes in melanoma. N Engl J Med. 2017;376(23):2280-1. 10.1056/NEJMe1704290. 
- Editorial article discussing the findings of DeCOG-SLT and MSLT-II arguing that regional control of non-sentinel lymph nodes is unrelated to distant metastasis, while producing minimal decreases in node-only recurrence.

[PubMed: 28591534]

37. Gershenwald JE, Andtbacka RH, Prieto VG, Johnson MM, Diwan AH, Lee JE, et al. Microscopic tumor burden in sentinel lymph nodes predicts synchronous nonsentinel lymph node involvement in patients with melanoma. J Clin Oncol. 2008;26(26):4296-303. 10.1200/JCO.2007.15.4179. [PubMed: 18606982]

38. Egger ME, Bower MR, Czyszczon IA, Farghaly H, Noyes RD, Reintgen DS, et al. Comparison of sentinel lymph node micrometastatic tumor burden measurements in melanoma. J Am Coll Surg. 2014;218(4):519-28. 10.1016/j.jamcollsurg.2013.12.014. [PubMed: 24491245]

39. van der Ploeg AP, van Akkooi AC, Haydu LE, Scolyer RA, Murali R, Verhoef C, et al. The prognostic significance of sentinel node tumour burden in melanoma patients: an international, multicenter study of 1539 sentinel node-positive melanoma patients. Eur J Cancer (Oxford, England : 1990). 2014;50(1):111-20. 10.1016/j.ejca.2013.08.023.

40. Kakavand H, Vilain RE, Wilmott JS, Burke H, Yearley JH, Thompson JF, et al. Tumor PD-L1 expression, immune cell correlates and PD-1+ lymphocytes in sentinel lymph node melanoma metastases. Mod Pathol. 2015;28(12):1535-44. 10.1038/modpathol.2015.110.

- Pathologic case series correlating lymphocyte subpopulations or PD-L1 expression in melanomapositive sentinel lymph node biopsy specimens with clinicopathologic features and outcome. Increased CD3+, CD4+, and CD8+ tumor-infiltrating lymphocytes correlated positively with recurrence-free survival, while peritumoral PD-1+ lymphocytes correlated negatively and PD-L1 expression did not correlate significantly.

[PubMed: 26403784]

41. Ryan M, Crow J, Kahmke R, Fisher SR, Su Z, Lee WT. FoxP3 and indoleamine 2,3-dioxygenase immunore-activity in sentinel nodes from melanoma patients. Am J Otolaryngol. 2014;35(6):68994. 10.1016/j.amjoto.2014.08.009. [PubMed: 25212103]

42. Speeckaert R, Vermaelen K, van Geel N, Autier P, Lambert J, Haspeslagh M, et al. Indoleamine 2,3-dioxygenase, a new prognostic marker in sentinel lymph nodes ofmelanoma patients. Eur $\mathbf{J}$ Cancer (Oxford, England : 1990). 2012;48(13):2004-11. 10.1016/j.ejca.2011.09.007.

43. Gershenwald JE, Scolyer RA, Hess KR, Sondak VK, Long GV, Ross MI, et al. Melanoma staging: evidence-based changes in the American joint committee on Cancer eighth edition cancer staging manual. CA Cancer J Clin. 2017;67(6):472-92. 10.3322/caac.21409. [PubMed: 29028110]

44. Bilimoria KY, Balch CM, Bentrem DJ, Talamonti MS, Ko CY, Lange JR et al. Complete lymph node dissection for sentinel node-positive melanoma: assessment ofpractice patterns in the United States. Ann Surg Oncol. 2008;15(6):1566-76. 10.1245/s10434-008-9885-2. [PubMed: 18414952]

45. Nocera NF, Pyfer BJ, De La Cruz LM, Chatterjee A, Thiruchelvam PT, Fisher CS. NSQIP analysis of axillary lymph node dissection rates for breast cancer: implications for resident and fellow participation. J Surg Educ. 2018 10.1016/j.jsurg.2018.02.020.

- Retrospective NSQIP (2007-2014) database analysis of Axillary Lymph Node Dissection Rates for Breast Cancer, which showed that rates of axillary lymph node dissection (ALND) for invasive breast cancer decreased $2.43 \%$ yearly from 2007 to 2014, with significant decreases observed in junior resident, but not senior resident and fellow participation.

46. Eggermont AM, Chiarion-Sileni V, Grob JJ, Dummer R, Wolchok JD, Schmidt H, et al. Prolonged survival in stage III melanoma with ipilimumab adjuvant therapy. N Engl J Med. 2016;375(19): 1845-55. 10.1056/NEJMoa1611299.

- Five-year follow-up for a phase 3, randomized controlled clinical trial in patients with resected stage III melanoma resection of stage III cutaneous melanoma, ipilimumab resulted in higher 
recurrence-free survival, overall survival, and distant metastasis-free survival than placebo, at the cost of increased grade 3 or 4 immune-related adverse events.

[PubMed: 27717298]

47. Eggermont AM, Chiarion-Sileni V, Grob JJ, Dummer R, Wolchok JD, Schmidt H, et al. Adjuvant ipilimumab versus placebo after complete resection of high-risk stage III melanoma (EORTC 18071): a randomised, double-blind, phase 3 trial. Lancet Oncol. 2015;16(5):522-30. 10.1016/ s1470-2045(15)70122-1.

- Phase 3, Double-blind, multi-center, randomized controlled clinical trial in patients with resected stage III cutaneous melanoma without previous systemic therapy, in which median and 3-year recurrence-free survival was higher in the ipilimumab compared to placebo group, though at a higher rate grade 3 or 4 adverse events.

[PubMed: 25840693]

48. Schachter J, Ribas A, Long GV, Arance A, Grob JJ, Mortier L, et al. Pembrolizumab versus ipilimumab for advanced melanoma: final overall survival results of a multicentre, randomised, open-label phase 3 study (KEYNOTE-006). Lancet. 2017;390(10105):1853-62. 10.1016/ S0140-6736(17)31601-X.

- A phase 3 randomized controlled clinical trial in patients with advanced melanoma, finding improved 1-year RFS for patients receiving pembrolizumab compared to ipilimumab.

[PubMed: 28822576]

49. Wolchok JD, Chiarion-Sileni V, Gonzalez R Rutkowski P, Grob JJ, Cowey CL, et al. Overall survival with combined nivolumab and ipilimumab in advanced melanoma. N Engl J Med. 2017;377(14):1345-56. 10.1056/NEJMoa1709684.

- 3-year follow-up of a phase 3 randomized controlled clinical trial in which combination therapy with nivolumab plus ipilimumab produced greater 3-year overall survival compared with nivolumab alone, which was in turn greater than in ipilimumab alone.

[PubMed: 28889792]

50. Long GV, Atkinson V, Cebon JS, Jameson MB, Fitzharris BM, McNeil CM, et al. Standard-dose pembrolizumab in combination with reduced-dose ipilimumab for patients with advanced melanoma (KEYNOTE-029): an open-label, phase 1b trial. Lancet Oncol. 2017;18(9):1202-10. 10.1016/s1470-2045(17)30428-x. [PubMed: 28729151] 


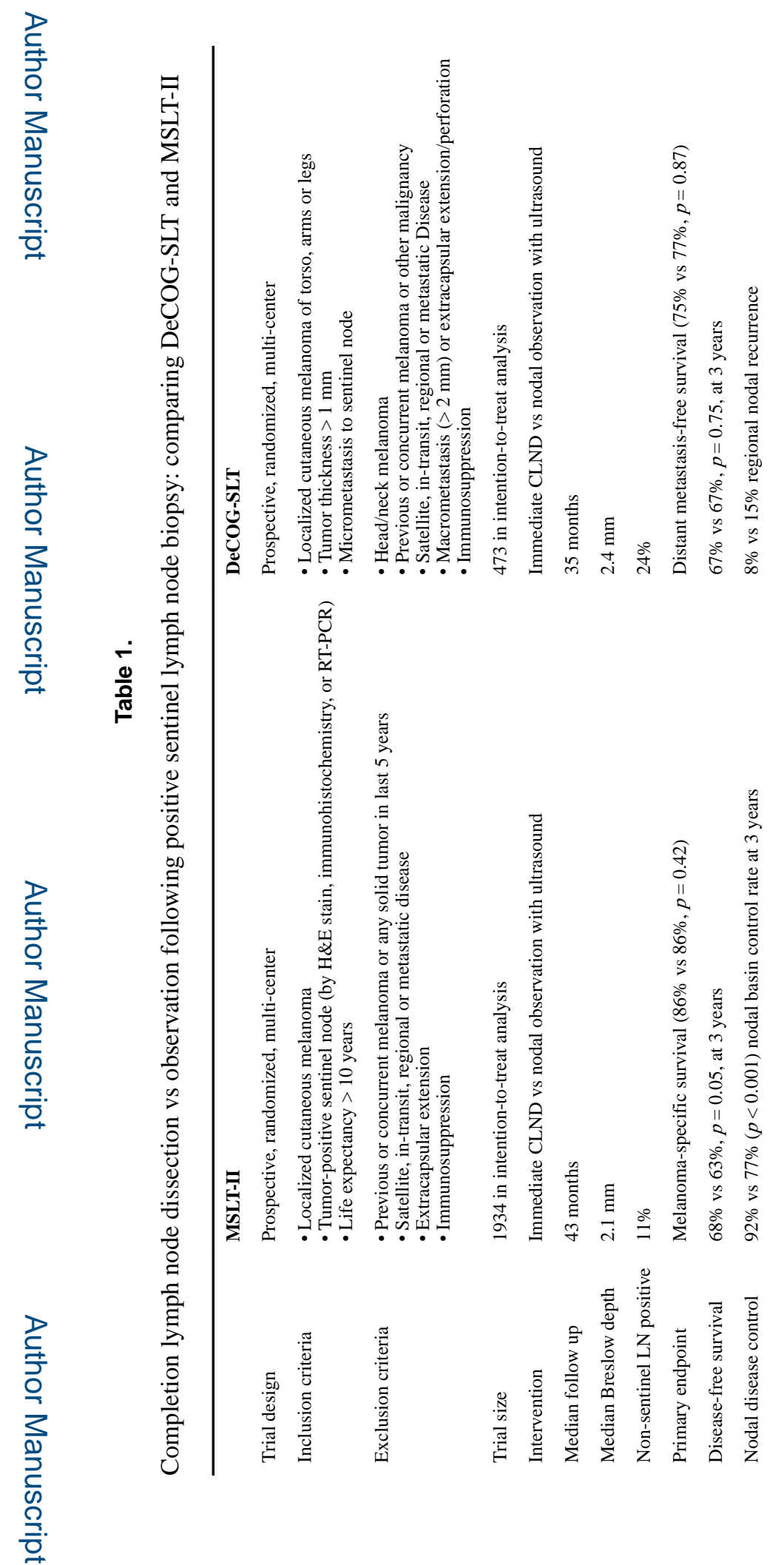

Curr Treat Options Oncol. Author manuscript; available in PMC 2019 August 06. 